

\title{
Effect of Distance for Chipless RFID Magnitude Coding
}

Olivier Rance, Etienne Perret, Romain Siragusa, Pierre Lemaitre-Auger

\section{To cite this version:}

Olivier Rance, Etienne Perret, Romain Siragusa, Pierre Lemaitre-Auger. Effect of Distance for Chipless RFID Magnitude Coding. IEEE Journal of Radio Frequency Identification, 2019, 3, 10.1109/JRFID.2019.2903386 . hal-02382196

\section{HAL Id: hal-02382196 https://hal.univ-grenoble-alpes.fr/hal-02382196}

Submitted on 27 Nov 2019

HAL is a multi-disciplinary open access archive for the deposit and dissemination of scientific research documents, whether they are published or not. The documents may come from teaching and research institutions in France or abroad, or from public or private research centers.
L'archive ouverte pluridisciplinaire HAL, est destinée au dépôt et à la diffusion de documents scientifiques de niveau recherche, publiés ou non, émanant des établissements d'enseignement et de recherche français ou étrangers, des laboratoires publics ou privés. 


\title{
Effect of Distance for Chipless RFID Magnitude Coding
}

\author{
Olivier Rance $^{\circledR}$, Etienne Perret ${ }^{\circledR}$, Senior Member, IEEE, Romain Siragusa, and Pierre Lemaître-Auger
}

\begin{abstract}
An implementation of the hybrid frequencymagnitude coding is presented. This technique can be applied to enhance coding capacity of already existing chipless radiofrequency identification tags without increase of complexity. The dependency of the magnitude from the tag-reader distance is discussed and a compensation technique is proposed. An increase of 2.6 bits per resonator is demonstrated from measures in office environment.
\end{abstract}

Index Terms-Chipless RFID tags, polarization, radar crosssections, radiofrequency identification, scatterer.

\section{INTRODUCTION}

$\mathbf{R}$ ADIOFREQUENCY identification (RFID) is a technology that has emerged in the 1940's. Although RFID is now used in many areas with new functionalities provided, the optical barcode remains the preferred candidate for large scale distribution (item tracking) due to its low cost and ease of use. A promising solution to reduce the unit price of a RFID tag is to remove the silicon chip.

As first consequence the read range is reduced as compared to conventional RFID due to the absence of load modulation method. A read range of $30 \mathrm{~cm}$ is generally a good practical value (comparable with low cost barcode readers) for measurement of chipless tags in real environment.

Another consequence of the chip suppression is the significant decrease in coding capacity. Indeed in absence of chip, all the information must be encoded directly in the shape of the tag. The most classic approach which provides the best coding density is called frequency position coding. This consists in using resonant elements whose resonant frequency can be set independently from one another. The information is carried by the presence or absence of peaks at specific frequencies of the signature (horizontal axis in Fig. 1). Coding capacities achieved in this way remain less than fifty bit [1]-[3] which is insufficient for most industrial applications. It is generally considered that chipless RFID should achieve a coding capacity of 128 bits for a tag with the size of a credit card for mass market penetration.

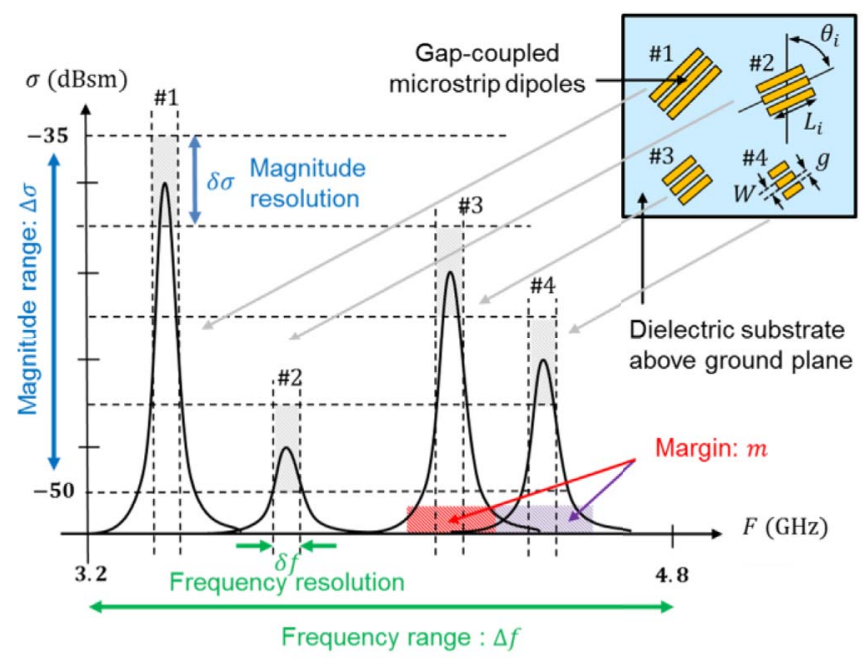

Fig. 1. RCS magnitude coding principle. The tag is composed of resonant scatterers which produce peaks in the scattered spectrum. The information is coded on both the frequency and the magnitude level of each scatterer.

To further increase the coding capacity, it is interesting to use a hybrid coding which combines the resonant frequency of the peak with another physical quantity, independent of the resonant frequency: the magnitude of the peak (vertical axis in Fig. 1).

The principle of "magnitude coding" has been first described in [4] for counterfeiting applications. The control of magnitude is realized with the presence or absence of a transparent resistive ink, giving different coding with the same visual aspect. This first occurrence was not particularly focused on coding capacity enhancement. Another implementation of magnitude coding for a tag without a ground plane has been studied in [5]. The magnitude control is done by coupling between adjacent resonators but this limits the increase of capacity ( +2 bits for the whole tag) as it becomes difficult to adjust independently the magnitude. Another approach to control the magnitude of resonant scatterers is to use the polarization mismatch between the tag and the reader [6]. This approach has the advantage of being applicable to any tag containing resonant elements.

An important result from the Singular Expansion Method (SEM) theory [7] is that unlike the natural resonance frequencies which are specific to the tag, the magnitude of the peaks depends both on the tag and on the measurement configuration, in particular on the distance between the tag and the reader. This makes magnitude coding particularly challenging, and the significant issue is not addressed in [4]-[6]. 


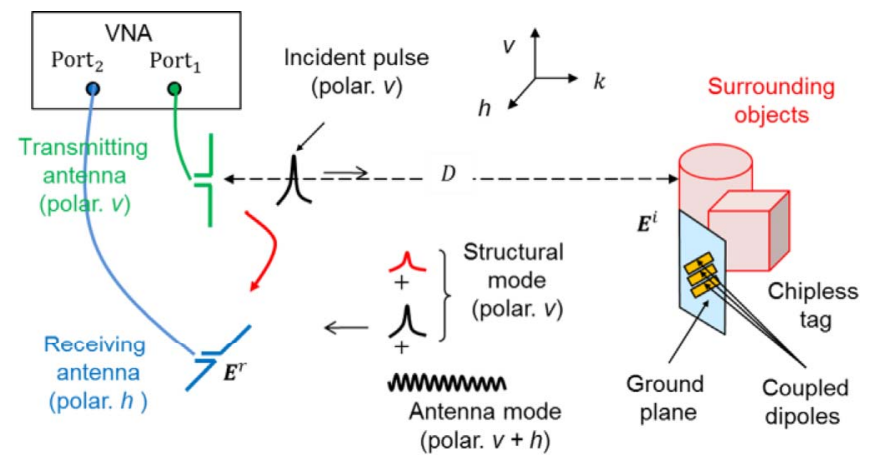

Fig. 2. Cross-polarization measurement setup. The structural mode is common to all scatterers and is a deformed copy of the incident pulse. The antenna mode is specific to the tag and has a different polarization than the incident pulse.

In this study, we propose to show how an already existing tag [8] originally designed for frequency coding can be re-used to realize an hybrid magnitude - frequency coding, without impact on the resonant frequencies and without adding additional complexity. We will also show that with a minimum calibration procedure, it is possible to recover the magnitude information of the tag by integrating calibration elements directly within the tag. Note that an extensive study concerning frequency coding has been done in [8]. The present article is thus focusing mainly on the magnitude aspect of the hybrid magnitude - frequency coding and only one frequency configuration showing the worst case for small frequency separation is shown.

The article is organized as follows: in part II we will present the tag geometry and the magnitude control technique. Part III focuses on the dependence to distance. Part IV is a discussion about the coding capacity offered by the hybrid method.

\section{TAg Geometry And Magnitude Control}

A number of different optimized chipless RFID tags are available in literature. To cross the fifty bits, it is becoming clear that playing on the shape of the tag or the value of some geometric parameter will not be sufficient and that new coding techniques are needed. From this statement, it seems relevant to use already optimized tags whose reading in real environments is particularly robust [8] and to adapt them for the implementation of an hybrid frequency-magnitude coding.

The detection principle of [8] is based on the use of depolarizing tags associated with a cross-polarization reading. The principle is recalled schematically in Fig. 2 but will not be discussed here as already extensively developed in [8]. Note that the cross polarization reading as well as the limited read range of $30 \mathrm{~cm}$ is reducing the potential effect of multipath which could be problematic for magnitude coding.

\section{A. Single Resonator}

The resonator used as RF encoding particle (REP) is the gap coupled dipole [8] which is represented schematically in the inline of Fig. 1. This REP has been optimized in terms of RCS, quality factor and surface. It is composed of $n=5$ microstrip dipoles of width $W=2 \mathrm{~mm}$, tightly coupled through a gap $g=0.5 \mathrm{~mm}$. The metallic strips are on top of a grounded Rogers RO4003C substrate having permittivity $\varepsilon_{r}=3.55$ and

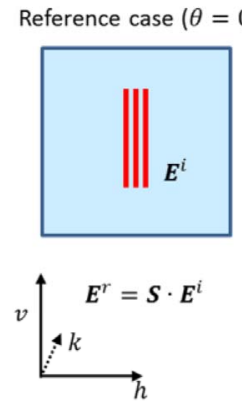

(a)

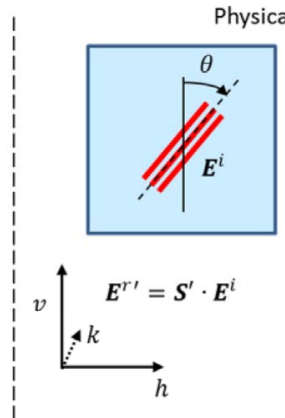

(b)

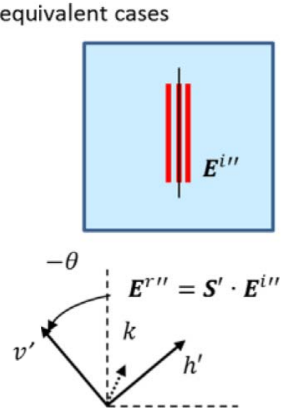

(c)
Fig. 3. Case studies. (a) Reference case. (b) Resonator rotated from an angle $\theta$ with respect to reference case. (c) Antennas rotated from and angle - $\theta$ with respect to reference case. The antennas are symbolically represented by their polarization vectors $h$ and $v$. Cases (b) and (c) are physically equivalent.

thickness $\mathrm{t}=0.8 \mathrm{~mm}$. The angle between the vertical orientation and the REP is $\theta$. The dipoles are initially oriented at $\theta=45^{\circ}$ to maximize the cross-polarization component of the response. As first approximation, gap-coupled microstrip dipoles can be considered as half-wavelength resonators [9]. The resonant frequency of the coupled microstrip dipoles is thus mainly set by the strips length $L$ which is a varying parameter. Note that to improve the RCS level multiple dipoles have been added.

\section{B. Magnitude Control}

The control of the tag backscattered wave magnitude is based on the polarization mismatch between the tag and the reader. The complete description of the interaction of the incident wave with the tag is given by the polarization scattering matrix $\boldsymbol{S}$, which relates the scattered electric field vector, $\boldsymbol{E}^{\boldsymbol{r}}$, to the incident field vector, $\boldsymbol{E}^{i}$ (see Fig. 2), component by component, [10]-[12]:

$$
\boldsymbol{E}^{r}=\left[\begin{array}{c}
E_{h}^{r} \\
E_{v}^{r}
\end{array}\right]=\left[\begin{array}{ll}
S_{h h} & S_{h v} \\
S_{v h} & S_{v v}
\end{array}\right] \cdot\left[\begin{array}{c}
E_{h}^{i} \\
E_{v}^{i}
\end{array}\right]=\boldsymbol{S} \cdot \boldsymbol{E}^{i}
$$

The quantity of interest is the cross-polarization component: $E_{h}^{r}=S_{h v} \cdot E_{v}^{i}$. Three case studies are examined. They are designated by (a), (b) and (c) on Fig. 3. The transmitting and receiving antenna are assumed to have a vertical and horizontal polarization respectively. They are symbolically represented by their polarization vectors $h$ and $v$ in Fig. 3. The scattering matrix of the reference case (a) is denoted by $\boldsymbol{S}$ (1). Now, we consider a rotation of angle $\theta$ of the resonator [case (b) in Fig. 3]. A new scattering matrix $\boldsymbol{S}^{\prime}$ is obtained:

$$
\boldsymbol{E}^{r \prime}=\boldsymbol{S}^{\prime} \cdot \boldsymbol{E}^{i}
$$

Physically, applying a rotation of angle $\theta$ to the resonator [case (b) in Fig. 3] is the same as applying a rotation of angle $-\theta$ to the antennas [case (c) in Fig. 3] such that the scattering matrix associated to case (c) is equal to $S^{\prime}$ :

$$
E^{r \prime \prime}=S^{\prime} \cdot E^{i \prime \prime}
$$

The incoming and reflected fields of case (c) are linked to the ones of case (a) by:

$$
\begin{aligned}
& \boldsymbol{E}^{i}=\boldsymbol{\Omega}^{T} \cdot \boldsymbol{E}^{i \prime \prime}, \\
& \boldsymbol{E}^{r}=\boldsymbol{\Omega}^{T} \cdot \boldsymbol{E}^{r \prime \prime},
\end{aligned}
$$


where $\boldsymbol{\Omega}^{T}$ is the transpose of the rotation matrix of angle $\theta$.

Injecting (4)-(5) into (1) and considering the fact that $\boldsymbol{\Omega}$ is a unitary matrix gives:

$$
\boldsymbol{E}^{r \prime \prime}=\boldsymbol{\Omega} \cdot \boldsymbol{S} \cdot \boldsymbol{\Omega}^{T} \cdot \boldsymbol{E}^{i \prime \prime} .
$$

By identification with (3), it finally comes:

$$
\boldsymbol{S}^{\prime}=\boldsymbol{\Omega} \cdot \boldsymbol{S} \cdot \boldsymbol{\Omega}^{T} \text {. }
$$

In this formulation, it is readily seen that the rotation angle $\theta$ can be used to modify the measured magnitude of the scattered field. The formula is valid for any kind of resonator and the method is then easy to adapt to other kind of REP. Considering the case of gap coupled dipoles, an interesting result is that for the reference angle $\theta=0$ (strips oriented along the vertical direction), the scattering matrix reduces to a simple form:

$$
\left[\begin{array}{c}
E_{h}^{r} \\
E_{v}^{r}
\end{array}\right]=\left[\begin{array}{cc}
0 & 0 \\
0 & S_{v v}(0)
\end{array}\right] \cdot\left[\begin{array}{c}
E_{h}^{i} \\
E_{v}^{i}
\end{array}\right] .
$$

Mathematically $S_{v v}$ and 0 are the eigenvalues of the scattering matrix function. Those particular quantities are considered to be particularly significant for target identification [11]. Expressing the measured quantity $S_{h v}$ relatively to this special case provides a particularly simple expression:

$$
\left|S_{h v}(\theta)\right|=\left|S_{v v}(0) \cdot \sin (2 \theta) / 2\right| .
$$

It is thus possible to control the signature magnitude value by varying $\theta$. The maximum cross-polarization value is obtained for $\theta=45^{\circ}$ and corresponds to $S_{v v}(0) / 2$. This coding technique implies the use of a specific reader able to change the incident field polarization while keeping a cross-polarization measurement configuration [13].

A series of measurements have been performed to validate the magnitude control of one REP included in the tag with other resonators, i.e., in presence of couplings. Four resonators having different length are positioned on a ground plane (inline of Fig. 1). Each resonator is realized individually in order to modify its orientation easily (inline photo of Fig. 4). The uncertainty due to manual placement of the resonators is evaluated to $\pm 1^{\circ}$. This uncertainty becomes important for $\theta$ approaching 0 which corresponds to maximum sensitivity of (9). The resonator number 3 is rotated such that the response is modified from theoretical magnitude steps of $1.5 \mathrm{~dB}$. The other resonators are kept in a constant position corresponding to their maximum magnitude $\left(\theta=45^{\circ}\right)$. Measurements (Fig. 4) are done in anechoic chamber, for a tag positioned at $20 \mathrm{~cm}$ from the reader. An empty measurement without tag $\left(\mathrm{E}_{21}\right.$ in the vertical axis of Fig. 4) is done to remove antenna couplings. A maximum deviation of $0.4 \mathrm{~dB}$ is observed for the resonator $n^{\circ} 4$ (immobile) which shows that the coupling induced from the rotated resonator has little influence. The peaks of resonator $n^{\circ} 3$ are decreasing with a regular $1.5 \mathrm{~dB}$ step as predicted in theory (indicated by grey bars in Fig. 4). For lower peak values, the decrease is less regular which can be due to positioning uncertainty. An overall magnitude range of $9 \mathrm{~dB}$ is obtained.

\section{EFFECT OF Distance}

The measurement setup is represented schematically in Fig. 2. As previously seen, in practice the quantity that can be measured directly is not the components of the scattering

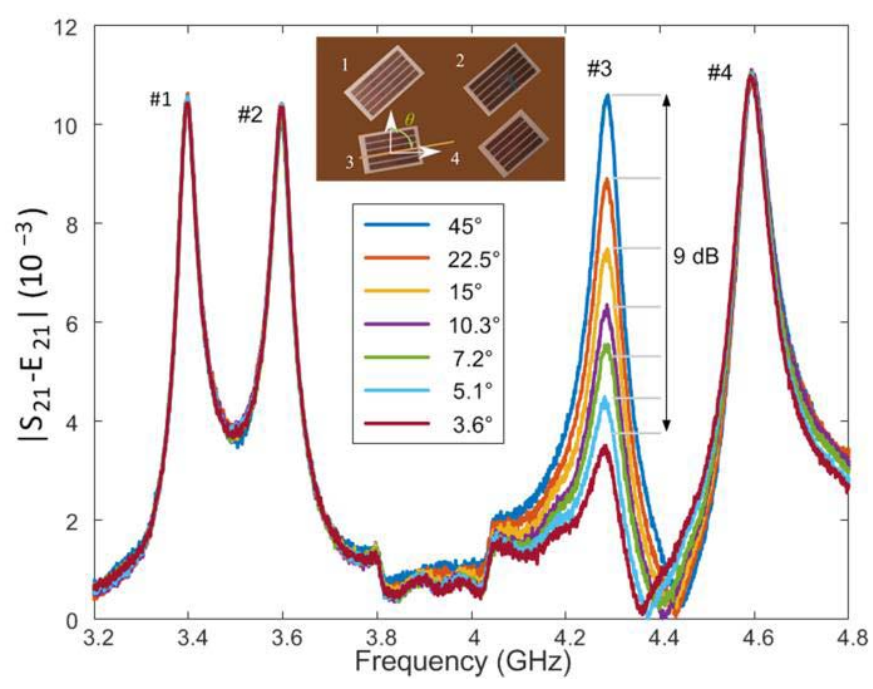

Fig. 4. Measured magnitude response of a tag with one scatterer rotated successively, in an anechoic chamber with a reading distance $\mathrm{D}=20 \mathrm{~cm}$. The length of the dipoles are $\mathrm{L}_{1}=23 \mathrm{~mm}, \mathrm{~L}_{2}=21.8 \mathrm{~mm}, \mathrm{~L}_{3}=17.9 \mathrm{~mm}$ and $\mathrm{L}_{4}=16.8 \mathrm{~mm}$.

matrix $S$ but the s-parameters of the VNA. Assuming that the VNA is calibrated at the antenna ports, the $S_{21}$ parameter of the VNA can be related to the components of the polarization scattering matrix $S$ with the monostatic radar equation [12]:

$$
S_{21}=\frac{G \cdot \lambda}{(2 \sqrt{\pi})^{3} \cdot D^{2}} S_{h v}
$$

where $G$ is the gain of the antenna and $\lambda$ is the wavelength in vacuum. In (10), it is clearly seen that the measured quantity $S_{21}$ depends on the reading distance $D$ and the antenna characteristics. As it can be seen from the measurement results of Fig. 5, the magnitude of the peak apex is changing with the distance D which may lead to false level detection.

\section{A. Compensation Technique}

A practical way to deal with the dependency of the response with respect to distance is to use one of the resonators as a calibration element directly integrated within the tag. We can consider as an example that we know the magnitude level of the first peak $S_{21}^{D_{r}}\left(f_{1}\right)$ for a reading distance of reference $D_{r}=20 \mathrm{~cm}$. Then the tag is measured at an unknown distance $D_{2}$ which gives the measured quantity $S_{21}^{D_{2}}$. Using the radar equation (10) for both measurements, it comes:

$$
S_{21}^{D_{2}}=\left(D_{r} / D_{2}\right)^{2} \cdot S_{21}^{D_{r}}
$$

As $S_{21}^{D_{r}}\left(f_{1}\right)$ is known, the ratio $D_{2} / D_{r}$ can be simply obtained from the evaluation of $S_{21} D_{2}$ at $f_{1}=3.4 \mathrm{GHz}$ (first peak). The broadband response of the tag reduced to the reference distance $D_{r}$ is then given by:

$$
S_{21}^{D_{2}}(f)=\left[\frac{S_{21}^{D_{2}}\left(f_{1}\right)}{S_{21}^{D_{r}}\left(f_{1}\right)}\right] \cdot S_{21}^{D_{r}}(f)
$$

For this approach, the magnitude value of the first peak must be the same from one tag to another and it cannot be used to code information anymore. In a certain manner, we can consider that the first resonator is a calibrating element. 


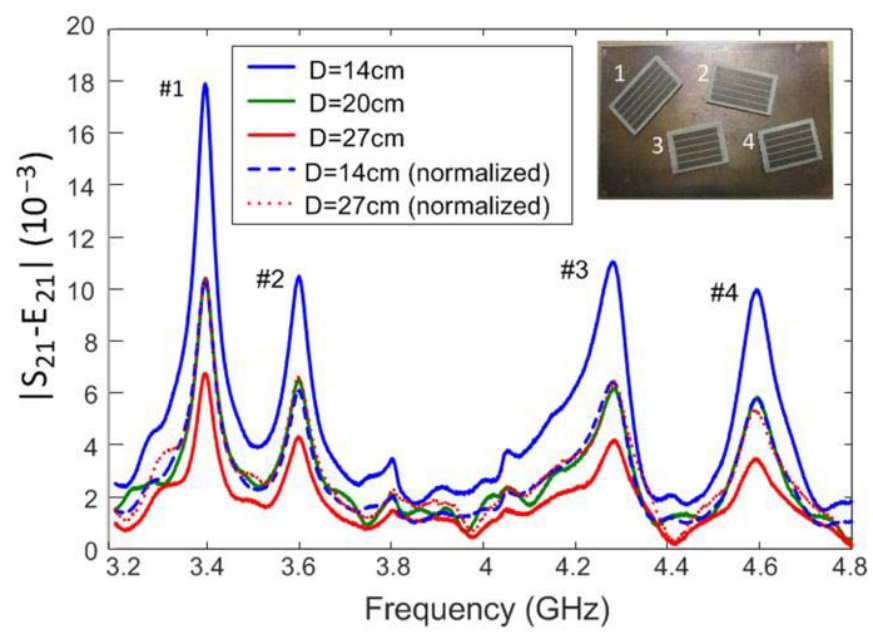

Fig. 5. Measured magnitude response of a tag with different reading distances. The normalized cases are calculated with (12) for the reference distance $D_{r}=20 \mathrm{~cm}$

Equation (12) shows that a reading error on the magnitude of the first resonator has repercussions on the other peaks. The first resonator is chosen as calibration element because it is the one having the highest quality factor. The orientation of the first resonator $\left(\theta=45^{\circ}\right)$ is also chosen to maximize magnitude in cross-polarization.

Then, assuming that the variation of the antenna gain with respect to frequency is known, it is possible to calculate $S_{v h}$ from the measured quantity $S_{21} D_{2}(f)$ by using the radar equation (10).

The response of the tag for different distances reduced to the reference case $D_{r}=20 \mathrm{~cm}$ (12) are compared in Fig. 5 . A maximum deviation of $0.7 \mathrm{~dB}$ appears for the peak $\mathrm{n}^{\circ} 4$. This value of $0.7 \mathrm{~dB}$ is doubled $(1.5 \mathrm{~dB})$ in order to define the magnitude resolution of the system (see Fig. 1). The magnitude resolution is defined in logarithmic scale to be independent to distance. Note that additional measurement have been done in anechoic chamber for read ranges up to $50 \mathrm{~cm}$ and would lead to a magnitude resolution of $3.2 \mathrm{~dB}$ following the same approach. However, the field magnitude obtained at this distance is probably too low for real environment applications.

\section{B. Measurement and Decoding of Predefined IDs}

A realistic magnitude resolution of $1.5 \mathrm{~dB}$ is chosen to evaluate the feasibility of the approach for a reading distance up to $27 \mathrm{~cm}$. The magnitude range of $9 \mathrm{~dB}$ considered for this approach allows 6 different magnitude levels for each scatterer. The code associated to a tag is composed of four digits $X_{1} X_{2} X_{3} X_{4}$, where $X_{i}$ corresponds the resonator number $i$. $X_{i}$ can vary between 0 and 5 where 0 identifies the level of the highest magnitude and 5 the level of the lowest magnitude.

Different IDs (0000, 0132, 0244 etc. see legend of Fig. 6) are chosen in advance and the resonators are rotated with the corresponding angle in the tag presented in the inline of Fig. 6 to realize those codes. Then the backscatter E-field of the tags is measured at $20 \mathrm{~cm}$. The objective is to verify that the IDs can be decoded from the measurement results with no error. The correspondence between the digit value, the magnitude slot and the value of the angle $\theta$ is given in table I.

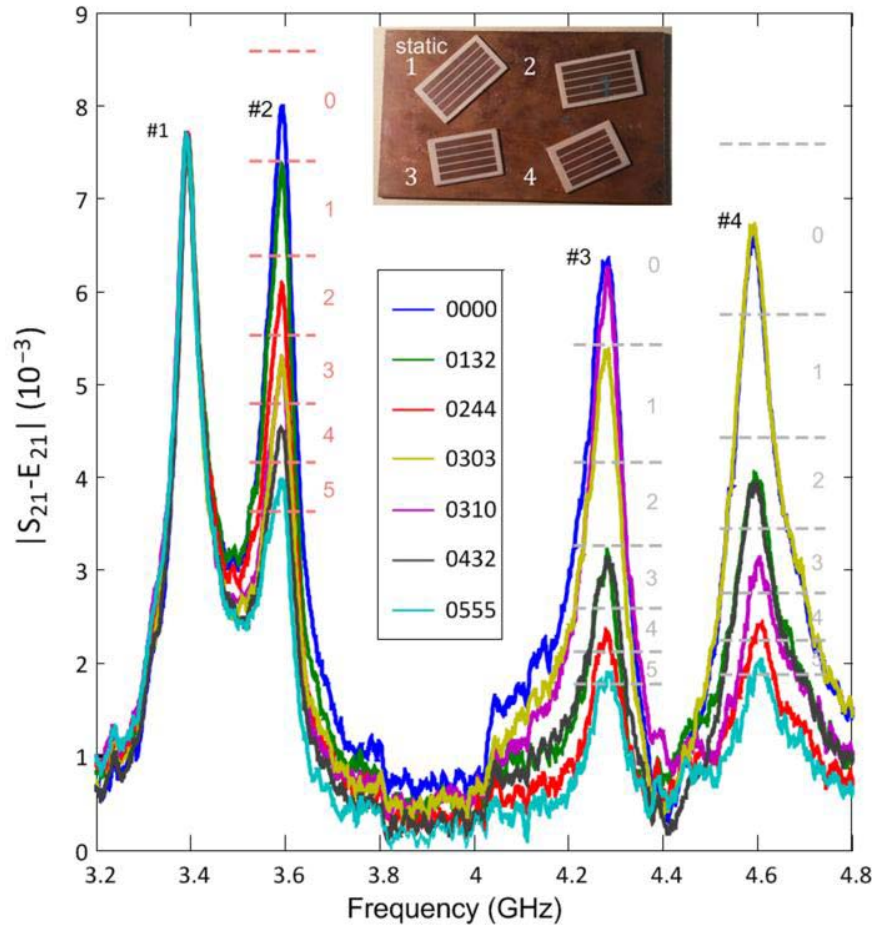

Fig. 6. Measurement results (office environment) for predefined IDs. The magnitude slots of $1.5 \mathrm{~dB}$ are indicated by grey bars in the figure. The first resonator is used as reference.

TABLE I

Correspondence Between Digit Value, ANGLE AND MAGNITUde SLOT

\begin{tabular}{|l|l|l|l|l|l|l|}
\hline Digit value & 0 & 1 & 2 & 3 & 4 & 5 \\
\hline Angle $\left(^{\circ}\right)$ & $45^{\circ}$ & 22.5 & 15 & 10.3 & 7.2 & 3.6 \\
\hline Magnitude & $+0.75 ;$ & $-0.75 ;$ & $-2.25 ;$ & $-3.75 ;$ & $-5.25 ;$ & $-6.75 ;$ \\
slot $(\mathrm{dB})$ & -0.75 & -2.25 & -3.75 & -5.25 & -6.75 & -8.25 \\
\hline
\end{tabular}

Measurements have been performed for the given IDs in real environment (office environment) with a dual polarization Satimo QH2000 antenna having a gain varying from $5 \mathrm{dBi}$ to $8 \mathrm{dBi}$ on the frequency band from $3 \mathrm{GHz}$ to $5 \mathrm{GHz}$. Measurements have been performed at a distance $\mathrm{D}=20 \mathrm{~cm}$ from the antennas. A Hamming time window is applied on the data acquired from the VNA. The lower limit of the Hamming window is taken at $8 \mathrm{~ns}$ to filter-out the response of the plate [14]. The maximum value of the window is taken at 20 ns which corresponds approximately to the extinction of the resonant modes. Time window allows limiting both potential multipath effects and parasitic reflections on the walls of the office.

The measurement results are presented in Fig. 6. The first resonator is used as a calibration element and is therefore not used for coding. The magnitude slots corresponding to a magnitude resolution of $1.5 \mathrm{~dB}$ are indicated by grey bars in the figure. They are calculated with respect to the maximum value of each peak (0000 measure in Fig. 6). For peaks $n^{\circ} 3-n^{\circ} 4$ for which there is no influence from other peaks (no coupling), a very good agreement between the theoretical $1.5 \mathrm{~dB}$ resolution and the measured values is observed. The $X_{3}$ and $X_{4}$ digits can be decoded without errors for all codes. 


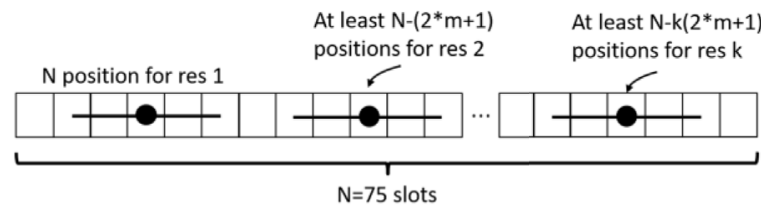

(a)

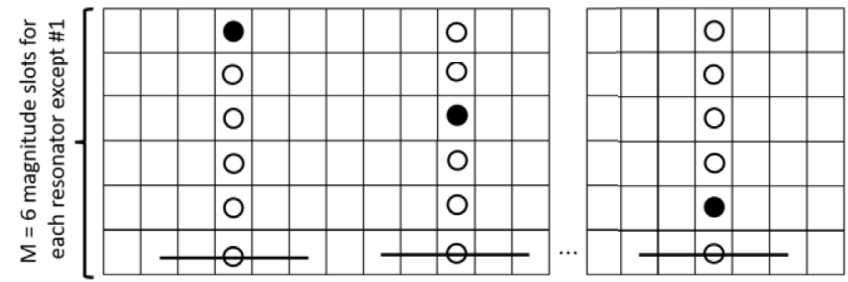

(b)

Fig. 7. Coding principle of (a) Frequency position coding and (b) Hybrid magnitude - frequency coding.

For the second digit $X_{2}$ (peak $\mathrm{n}^{\circ} 2$ ), the remaining contribution of the first peak disrupts and makes the decoding a bit more difficult. This contribution is additive and constant and arises because the frequency separation between the first two peaks $(200 \mathrm{MHz})$ is low which illustrates the worst case for the frequency configuration. However, it is possible to compensate this additive contribution. Indeed, for hybrid frequency-magnitude coding, first each peak frequency can be extracted from the backscattered signal, and depending on peak frequency shift between resonance frequencies, a compensation value can be used for decoding the information encoded in magnitude. In that example, the evaluation of the remaining contribution of the first peak at the frequency of the second peak gives a value of $3.1 \times 10^{-3}$ which is added to the theoretical response of peak $n^{\circ} 2$ for the angles given in table I. The calculated magnitude slots are then represented by red bars in Fig. 6. Using those red bars effectively gives a decoding with no error for the second digit $X_{2}$. This problem of interference between contiguous peaks can also be addressed simply by considering larger frequency spacing (300 MHz) between neighboring resonators.

From practical consideration, the tag orientation during the reading can be considered to be unknown. This additional constraint can be taken into consideration by using a specific reader able to change the incident field polarization while keeping a cross-polarization measurement configuration [13]. With such functionality, from the reader side, the orientation corresponding to the maximum of the peak apex $n^{\circ} 1$ can be determined. From the measurement at the specific orientation, the approach introduced here can be directly implemented.

\section{CODING CAPACITY}

In the present section, the evaluation of the coding capacity offered by the frequency coding is first considered. Then the additional capacity given by the hybrid magnitude - frequency coding is calculated in a second step.

The number of resonators is denoted by $n$. In the present case, $n=4$ resonators are considered. The four resonant peaks are spread over the UWB $(3.1 \mathrm{GHz}$ to $10.6 \mathrm{GHz})$ which provide an operating frequency band of $\Delta F=7.5 \mathrm{GHz}$. Based on the selectivity that can be achieved for the resonators, the

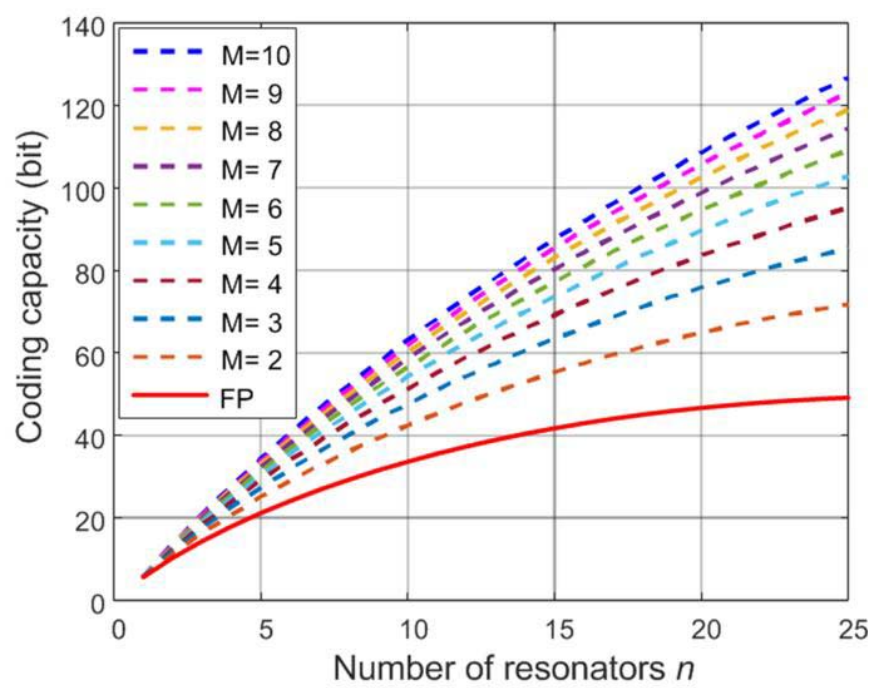

Fig. 8. Comparison between coding capacities of the frequency position coding alone (FP) and the hybrid magnitude - frequency position coding with respect to the number of resonators $n$ for different number of magnitude levels $M$. The hypothetical case of $N=50$ frequency slots is taken.

band is divided in frequency slots of length $\delta f=100 \mathrm{MHz}$. This gives a number of $N=\Delta f / \delta f=75$ potential slots for each resonator. We consider that a minimum frequency separation of $300 \mathrm{MHz}$ (see Fig. 7) is taken to limit the problem of neighboring contribution discussed in previous section. Each resonator has then to be separated from at least $m=2$ slots from its neighbor as represented schematically in Fig. 7-a. The total number of IDs is cumbersome to calculate due to the $300 \mathrm{MHz}$ separation but a close minorant is given by:

$$
C_{F P}=\frac{1}{n !} \cdot \prod_{k=1}^{n} N-k(2 \cdot m+1)
$$

For $n=4$ and $N=75$, this gives a total coding capacity of 19.7 bits for the present tag.

We consider now that in addition to the conventional frequency position coding, each resonator is coding $M=6$ magnitude levels except for the first one used as a calibration element. The hybrid magnitude-frequency coding principle is represented graphically in Fig. 7-b. For each frequency ID, there is then $M^{(k-1)}$ different possibilities related to the magnitude. The total number of IDs for the hybrid magnitude frequency position coding is then given by:

$$
C_{M C}=M^{(k-1)} \cdot \frac{1}{n !} \cdot \prod_{k=1}^{n} N-k(2 \cdot m+1)
$$

which gives a total coding capacity of 27.5 bits calculated from (14). An additional coding capacity of 2.6 bits per resonator (the first one is used as reference) is thus gained with the magnitude coding approach.

More generally, the comparison between the coding capacities of the two approaches can be done using (13) and (14). The hypothetical case of $N=50$ frequency slots is being considered. The coding capacity is represented in Fig. 8 with respect to the number of resonators $n$ for a various number of magnitude levels $M$. We see that magnitude coding makes a big improvement of the coding capacity, even for low number of resonators and low number of levels. 


\section{CONCLUSION}

A comprehensive study has been performed to examine the feasibility and the potential gain of the magnitude level coding for chipless RFID. The control of the magnitude is realized with the polarization mismatch between the tag and the antennas. This approach is simple to implement in practice because it implies only a rotation of the resonators and can be easily adapted to already optimized REPs. The initial tag consisting in four resonators was coded with the frequency position method for a capacity of 24.2 bits. The approach allowed increasing the coding capacity by 7.8 bits. It has been shown that the presence of a reference scatterer included within the tag allows measurement with a minimalist calibration process without to suffer from dependency to reading distance.

\section{REFERENCES}

[1] S. Preradovic and N. C. Karmakar, "Design of fully printable planar chipless RFID transponder with 35-bit data capacity," in Proc. Eur. Microw. Conf. (EuMC), Rome, Italy, 2009, pp. 013-016.

[2] A. Vena, E. Perret, and S. Tedjini, "High-capacity chipless RFID tag insensitive to the polarization," IEEE Trans. Antennas Propag., vol. 60, no. 10, pp. 4509-4515, Oct. 2012.

[3] R. Rezaiesarlak and M. Manteghi, "Complex-natural-resonance-based design of chipless RFID tag for high-density data," IEEE Trans. Antennas Propag., vol. 62, no. 2, pp. 898-904, Feb. 2014.

[4] A. Vena, A. A. Babar, L. Sydänheimo, M. M. Tentzeris, and L. Ukkonen, "A novel near-transparent ASK-reconfigurable inkjet-printed chipless RFID tag," IEEE Antennas Wireless Propag. Lett., vol. 12, pp. 753-756, 2013.

[5] O. Rance, R. Siragusa, P. Lemaître-Auger, and E. Perret, "Toward RCS magnitude level coding for chipless RFID," IEEE Trans. Microw. Theory Tech., vol. 64, no. 7, pp. 2315-2325, Jul. 2016.

[6] O. Rance, R. Siragusa, P. Lemaître-Auger, and E. Perret, "RCS magnitude coding for chipless RFID based on depolarizing tag," in Proc. IEEE MTT-S Int. Microw. Symp. (IMS), Phoenix, AZ, USA, 2015, pp. 1-4.

[7] C. E. Baum, E. J. Rothwell, K.-M. Chen, and D. P. Nyquist, "The singularity expansion method and its application to target identification," Proc. IEEE, vol. 79, no. 10, pp. 1481-1492, Oct. 1991.

[8] A. Vena, E. Perret, and S. Tedjni, "A depolarizing chipless RFID tag for robust detection and its FCC compliant UWB reading system," IEEE Trans. Microw. Theory Tech., vol. 61, no. 8, pp. 2982-2994, Aug. 2013.

[9] Antenna Theory: Analysis and Design, 3rd ed. Hoboken, NJ, USA: Wiley-Intersci., 2005.

[10] S. Riegger and W. Wiesbeck, "Wide-band polarimetry and complex radar cross section signatures," Proc. IEEE, vol. 77, no. 5, pp. 649-658, May 1989.

[11] J. R. Huynen, "Measurement of the target scattering matrix," Proc. IEEE, vol. 53, no. 8, pp. 936-946, Aug. 1965.

[12] E. F. Knott, J. F. Shaeffer, and M. T. Tuley, Radar Cross Section. Dedham, MA, USA: Artech House, 1985.

[13] M. Garbati, A. Ramos, R. Siragusa, E. Perret, and C. Halopé, "Chipless RFID reading system independent of polarization," in Proc. IEEE MTT-S Int. Microw. Symp. (IMS), San Francisco, CA, USA, 2016, pp. 1-3.

[14] A. Ramos et al., "Temporal separation detection for chipless depolarizing frequency-coded RFID," IEEE Trans. Microw. Theory Tech., vol. 64, no. 7, pp. 2326-2337, Jul. 2016.

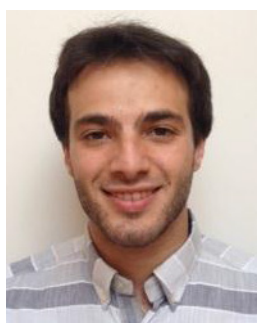

Olivier Rance received the M.Sc. and Ph.D. degrees in electrical engineering from the Institute National Polytechnique de Grenoble in 2012 and 2017, respectively. He is a Post-Doctoral Fellow with Telecom ParisTech. His current scientific interests include leaky-wave antennas, chipless RFID, and RF absorbers.

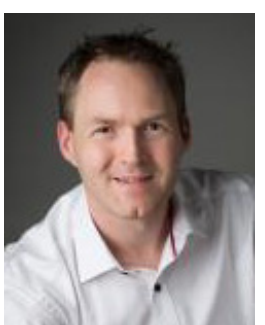

Etienne Perret (S'02-M'06-SM'13) received the Eng.Dipl. degree in electrical engineering from the Ecole Nationale Supérieure d'Electronique, d'Electrotechnique, d'Informatique, d'Hydraulique, et des Télécommunications, Toulouse, France, in 2002 and the M.Sc. and Ph.D. degrees in electrical engineering from the Toulouse Institute of Technology, Toulouse, in 2002 and 2005, respectively. From 2005 to 2006, he was a Post-Doctoral Fellow with the Institute of Fundamental Electronics, Orsay, France. Since 2006, he has been an Associate Professor of electrical engineering with the University of Grenoble Alpes, Grenoble Institute of Technology, Grenoble, France. Since 2014, he has been a Junior Member with the Institut Universitaire de France, Paris, France, an institution that Distinguishes Professors for their research excellence, as evidenced by their international recognition. Since 2015, he has been an Appointed Member of the French National Council of Universities. He has authored or co-authored over 180 technical conferences, letters, and journal papers, and books and book chapters. He holds several patents. His works have generated about 2000 citations. His current research interests include electromagnetic modeling of passive devices for millimeter and submillimeter-wave applications, and wireless communications, especially RFID and chipless RFID, and also include advanced computer-aided design techniques based on the development of an automated codesign synthesis computational approach. He was a recipient of the French Innovative Techniques for the Environment Award in 2013, the SEE/IEEE Leon Brillouin Award for his outstanding achievement in the identification of an object in an unknown environment using a chipless label or tag in 2016, the IEEE MTT-S 2019 Outstanding Young Engineer Award, and the ERC Consolidator Grant for his project ScattererID in 2017. He was named one of the MIT Technology Review's French Innovator's under 35 for his work on chipless RFID in 2013. He is a Technical Program Committee Member of the IEEE International Conference on RFID. He was a Keynote Speaker and the chairman of several international symposiums.

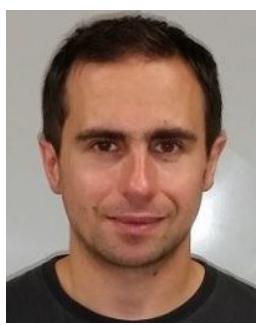

Romain Siragusa was born in Paris, France. He received the engineering and master's degrees from the Institute National Polytechnique de Grenoble (Grenoble INP) in 2006 and the Ph.D. degree from the Laboratoire de Conception et d'Intégration des Systèmes (LCIS), Grenoble INP, Valence, France. In 2010, he joined the Commissariat à l'Énergie Atomique as a Post-Doctoral Fellow, where he studied the high impedance surface for integrated antenna on silicon. Since 2011, he has been an Associate Professor with LCIS, Grenoble INP Esisar. His current scientific interests include leaky-wave structures and chipless RFID.

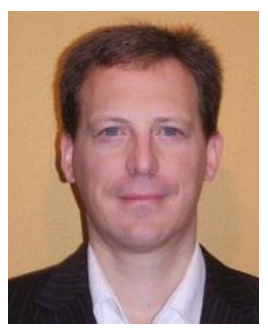

Pierre Lemaître-Auger received the B.Ing. and M.Sc.A. degrees in physical engineering from the École Polytechnique de Montréal, Canada, in 1992 and 1994, respectively, and the Doctor degree in optoelectronics and microwave from the Institut National Polytechnique de Grenoble, France, in 1998. In 1998, he joined the Engineering School, LCIS, Grenoble INP-Esisar, as an Associate Professor, where he participated to the creation of the Engineering School, especially to the elaboration of its teaching program in physics, and the Director of Physical Department from 1997 to 2006 and the Director of Study from 2006 to 2009. In 2010 and 2011, he was a Visiting Professor with the PolyGrames Microwave Research Center, École Polytechnique de Montréal. Since 2014, he has been the Head of International Relations of Grenoble INP-Esisar. His first research topics concerned integrated optics on glass substrates for sensing applications. From 2004 to 2010, he actively participated to the creation of a French startup company specialized in optical sensors. In 2006, he reoriented his research activities to radiofrequency. He has authored and co-authored over 100 journal papers, letters, or international conferences. He holds five patents. He now works on radiofrequency wave generation and propagation: antennas, array antennas, frequency selective surface, localized waves, and sensing. He was a recipient of several French awards for his research on wall-paper blocking WiFi signals. 